\title{
1-(2-) Prime Ideals in Semirings
}

\author{
PANDARINATHAN NANDAKUMAR \\ Department of Mathematics, Perunthalaivar Kamarajar Institute of Engineering \\ and Technology, (Government of Puducherry Institution), Nedungadu, Karaikal - \\ 609603 (U. T. of Puducherry), India \\ e-mail : drpnandakumar@gmail.com
}

ABSTRACT. In this paper, we introduce the concepts of 1-prime ideals and 2-prime ideals in semirings. We have also introduced $m_{1}$-system and $m_{2}$-system in semiring. We have shown that if $Q$ is an ideal in the semiring $R$ and if $M$ is an $m_{2}$-system of $R$ such that $\bar{Q} \bigcap M=\emptyset$ then there exists as 2-prime ideal $P$ of $R$ such that $Q \subseteq P$ with $P \bigcap M=\emptyset$.

\section{Introduction}

A semiring is a non-empty set $R$ equipped with two binary opertions, called addition, +, and multiplication (denoted by juxtaposition), such that $R$ is multiplicatively a semigroup and additively a commutative semigroup and that the multiplication is distributed across the addition both from the left and from the right. An element denoted by 0 is called the zero of $R$ if $a+0=a$ and $0 a=a 0=0$ for all $a \in R$. A non-empty subset of a semiring $R$ is called an ideal of $R$ iff $a+b \in I$, $r a \in I$, ar $\in I$ hold for all $a, b \in I$ and for all $r \in R$. The notions of left, right and two-sided ideals, as well as sums and products of such ideals are defined as usual. The word ideal will always mean a two-sided ideal. An ideal $I$ of $R$ is called a $k$-ideal if $a, a+b \in I$ implies $b \in I$ for any elements of $a, b \in R$. If $A$ is an ideal of a semiring $R$ then $\bar{A}=\{a \in R / a+x \in A$, for some $x \in A\}$ is called a $k$-closure of $A$. It can be easily verified that $\bar{A}$ is a $k$-ideal (see [6]). If $A \subseteq R$, then the ideal (k-ideal) of $R$ generated by $A$ will be denoted $\left\langle A>\left(\langle A\rangle_{k}\right)\right.$. If $A=a$, we write $\langle a\rangle$ instead of $\langle\{a\}\rangle$ for convenience. In this paper we introduce the concepts of $1-(2-)$ prime ideals as well as $1-(2-)$ semiprime ideals. If $R$ is semiring and $P$ is an ideal of $R$ then $P$ is $0-(2-)$ prime ideal if $A$ and $B$ are ideals ( $k$-ideals) of $R$ such that $A B \subseteq P$ implies $A \subseteq P$ or $B \subseteq P$. If $R$ is a semiring and $P$ is an ideal of $R$ then $P$ is 1-prime ideal if $A$ is a $k$-ideal of $R$ and $B$ is an ideal of $R$ such that $A B \subseteq P$ implies $A \subseteq P$ or $B \subseteq P$. If $R$ is a semiring and $Q$ is an ideal of $R$, then $Q$ is $0-(2-)$ semiprime ideal if $A$ is an ideal (k-ideal) of $R$ such that $A^{2} \subseteq Q$ implies $A \subseteq Q$. If $R$ is a semiring and $Q$ is an ideal of $R$ then $Q$ is 1-semiprime if $A$ is an ideal of $R$ such that $\bar{A} A \subseteq Q$ implies $A \subseteq Q$. A semiring $R$ is called fully idempotent if $I^{2}=I$ for every ideal $I$ of $R$.

Received April 25, 2009; accepted 10 September, 2009.

2000 Mathematics Subject Classification: 16Y60.

Key words and phrases: Semirings, Prime ideal, Semiprime ideal. 
If $A, B \subseteq R$, then we define $(A: B)_{l}=\{r \in R / r B \subseteq A\},(A: B)_{r}=\{r \in R / B r \subseteq$ $A\},(A: B)=\{r \in R / r B \subseteq A$ and $B r \subseteq A\}$, As easily seen, 0-prime ideal of $R$ is 2 -prime ideal of $R$, but not conversely. Clearly 0 -prime $\Longrightarrow 1$-prime $\Longrightarrow 2$-prime, 0-semiprime $\Longrightarrow 1$-semiprime $\Longrightarrow 2$-semiprime. Next we introduce the concepts of $m_{1}$-system, $m_{2}$-system, $n_{1}$-system and $n_{2}$-system. We have shown that if $Q$ is an ideal of $R$ and if $M$ is an $m_{2}$-system of $R$ such that $\bar{Q} \cap M=\emptyset$, then there exists a 2- prime ideal $P$ of $R$ such that $Q \subseteq P$ with $P \cap M=\emptyset$. Throughout this paper $R$ stands for semiring.

Definition 1.1. A semiring $R$ is an ordered triple $R=(R,+,$.$) such that (a)$ $<R,+>$ is a commutative monoid with identity denoted $0_{R}$ or simply 0 , (b) $<R$, . $>$ is a semigroup, (c) For every $r, s, t \in R, r(s+t)=r s+r t$ and $(s+t) r=s r+t r,(\mathrm{~d})$ For every $r \in R, r 0=0 r=0$.

Definition 1.2. Following Alarcon and Polkowska [2], we have the following definition for $\mathrm{B}(\mathrm{n}, \mathrm{i})$ semirings.

Let $n \geq 2 \in N$ and $0 \leq i<n$ and $m=n-i$. Let $B(n, i)$ be the following semirings :

$B(n, i)=\{0,1,2, \ldots, n-1\}$ and the operations in $B(n, i)$ are:

$$
\begin{aligned}
x+{ }_{B(n, i)} y= & \begin{cases}x+y & \text { if } x+y \leq n-1 \\
l & \text { if } x+y \geq n \\
\text { with } & l=(\mathrm{x}+\mathrm{y}) \bmod \mathrm{m} \text { and } i \leq l \leq n-1\end{cases} \\
x \cdot B(n, i) & = \begin{cases}x y & \text { if } x y \leq n-1 \\
l & \text { if } x y \geq n \\
\text { with } & l=(\mathrm{xy}) \bmod \mathrm{m} \text { and } i \leq l \leq n-1\end{cases}
\end{aligned}
$$

Definition 1.3. If $R$ is a semiring and $P$ is an ideal of $R$, then $P$ is $0-(2-)$ prime if $A$ and $B$ are ideals (k-ideals) of $R$ such that $A B \subseteq P$ implies $A \subseteq P$ or $B \subseteq P$. If $R$ is a semiring and $P$ is an ideal of $R$ then $P$ is 1-prime if $A$ is a k-ideal of $R$ and $B$ is an ideal of $R$ such that $A B \subseteq P$ implies $A \subseteq P$ or $B \subseteq P$.

Remark 1.4. As in rings, if $P$ is an ideal in a semiring $R$, then $P$ is a 0 -prime ideal iff $a, b \in R$ such that $a R b \subseteq P$ then $a \in P$ or $b \in P$.

As in rings, if $Q$ is an ideal in a semiring $R$, then $Q$ is a 0 -semiprime ideal iff $a \in R$ such that $a R a \subseteq Q$ then $a \in Q$.

Lemma 1.5. If $A$ and $B$ are left ideals of $R$ then $(A: B)_{l}$ is an ideal.

Lemma 1.6. If $A$ is a left $k$-ideal of $R$ and $B$ is a left ideal then $(A: B)_{l}$ is a 
$k$-ideal.

Lemma 1.7. If $A$ and $B$ are right ideals of $R$ then $(A: B)_{r}$ is an ideal.

Lemma 1.8. If $A$ is a right $k$-ideal of $R$ and $B$ is a right ideal then $(A: B)_{r}$ is a $k$-ideal.

Lemma 1.9. If $P$ is a 0 -prime ideal of $R$ then $P$ is a 2-prime ideal (1-prime ideal) of $R$.

But the converse need not be true as the folloiwng example shows.

Example 1.10. Consider the semiring $B(4,3)=\{0,1,2,3\}$, where + and . are defined as follows.

\begin{tabular}{c|cccc}
+ & 0 & 1 & 2 & 3 \\
\hline 0 & 0 & 1 & 2 & 3 \\
1 & 1 & 2 & 3 & 3 \\
2 & 2 & 3 & 3 & 3 \\
3 & 3 & 3 & 3 & 3 \\
$\cdot$ & 0 & 1 & 2 & 3 \\
\hline 0 & 0 & 0 & 0 & 0 \\
1 & 0 & 1 & 2 & 3 \\
2 & 0 & 2 & 3 & 3 \\
3 & 0 & 3 & 3 & 3
\end{tabular}

Since the ideals are $\{0\},\{0,3\},\{0,2,3\},\{0,1,2,3\}$ and $k$-ideals are $\{0\}$ and $\{0$, $1,2,3\}$ the ideal $\{0,3\}$ is 2 -prime but not 0 -prime.

Theorem 1.11. If $P$ is a $k$-ideal of $R$ then $P$ is a 0 -prime ideal if and only if $P$ is 2-prime ideal.

Proof. Let us assume that $P$ is 2-prime and $P$ is a k-ideal of $R$. Let us assume that $A$ and $B$ are ideals of $R$ such that $A B \subseteq P$. If $A B \subseteq P$, then $A \subseteq(P: B)_{l}$. By Lemma 1.6, $(P: B)_{l}$ is a $k$-ideal of $R$. Therefore $\left\langle A>_{k} \subseteq(P: B)_{l}\right.$. It follows that $<A>_{k} B \subseteq P$. Hence $B \subseteq\left(P:<A>_{k}\right)_{r}$. By Lemma 1.8, $\left(P:<A>_{k}\right)_{r}$ is a $k$-ideal of $R$. Therefore $<B>_{k} \subseteq\left(P:<A>_{k}\right)_{r}$. It follows that $<A>_{k}<B>_{k} \subseteq P$ Since $P$ is 2-prime, we have $<A>_{k} \subseteq P$ or $<B>_{k} \subseteq P$. Hence $A \subseteq P$ or $B \subseteq P$.

Definition 1.12. $M \subseteq R$ is called an $m_{0}$-system if for every $a, b \in M$ there exists $x \in R$ such that $a x b \in M . M \subseteq R$ is called an $m_{1}$-system if for every $a, b \in M$ there exists $a_{1} \in\langle a\rangle_{k}$ and there exists $b_{1} \in\langle b\rangle$ such that $a_{1} b_{1} \in M . M \subseteq R$ is called an $m_{2}$ system if for every $a, b \in M$ there exists $\left.a_{1} \in<a\right\rangle_{k}$ and there exists $b_{1} \in\langle b\rangle_{k}$ such that $a_{1} b_{1} \in M$.

Lemma 1.13. Every $m_{0}$ system is an $m_{2}$ ( $m_{1}$-system $)$. But the converse need not 
be true as the following example shows.

Example 1.14. Consider the semiring $\mathrm{B}(4,3)$ in Example 1.10. Clearly $M=$ $\{1,2\}$ is an $m_{2}$ system, but not an $m_{0}$-system.

Lemma 1.15. If $P$ is an ideal of $R, P$ is a 2-prime ideal (1-prime ideal, 0-prime ideal) of $R$ iff $R \backslash P$ is an $m_{2}$-system ( $m_{1}$ system, $m_{0}$-system) of $R$.

Theorem 1.16. Let $Q$ be an ideal of $R$, and let $M$ be an $m_{2}$-system ( $m_{1}$-system) of $R$ such that $\bar{Q} \cap M=\emptyset$. Then there exists a 2-prime ideal (1-prime ideal) $P$ of $R$ such that $Q \subseteq P$ with $P \cap M=\emptyset$.

Proof. Let $Q$ be an ideal of $R$ and let $M$ be an $m_{2}$-system of $R$ such that $\bar{Q} \cap M=\emptyset$. Now we consider the set $\mathcal{M}=\{I /$ (i) $I$ is an ideal of $R$ such that $Q \subseteq I$, (ii) $\bar{I} \cap M=\emptyset\}$. Clearly $Q$ is in $M$. Let $Q_{1} \subseteq Q_{2} \subseteq Q_{3} \subseteq \ldots$ be any chain of ideals of $\mathcal{M}$. Let $A=\bigcup Q_{i}$. Clearly $A$ is an ideal of $R$. We claim that $\bar{A} \cap M=\emptyset$. Suppose not, let $a \in \bar{A} \cap M$ implies $a \in \bar{A}$ and $a \in M$. Hence $a+x \in A$ for some $x \in A$. Since $a+x \in A=\bigcup Q_{i}$ implies $a+x \in Q_{i}$ for some $i$, since $x \in A=\cup Q_{i}$ implies $x \in Q_{k}$ for some $k$. Without loss of generality let us assume that $k<i$. Then $a+x, x \in Q_{i}$ implies $a \in \overline{Q_{i}}$. Therefore $a \in \overline{Q_{i}} \cap M$ which is a contradiction, since $\overline{Q_{i}} \cap M=\emptyset$. Thus $\bar{A} \cap M=\emptyset$. Then by Zorn's Lemma, there exists an ideal $P$ of $R$ that is maximal with respect to above properties. Let $A$ be a $k$-ideal of $R$ such that $A \nsubseteq P$. We calim that $(\bar{P}: A)_{r}=(\bar{P}: A)_{l}=P$. Since $P$ is an ideal we have $A P \subseteq P \subseteq \bar{P}$ and so $P \subseteq(\bar{P}: A)_{r}$. Similarly $P \subseteq(\bar{P}: A)_{l}$. By Lemma 1.8, $(\bar{P}: A)_{r}$ is a $k$-ideal and by Lemma $1.6(\bar{P}: A)_{l}$ is a $k$-ideal. Thus $(\bar{P}: A)_{l}$ and $(\bar{P}: A)_{r}$ are $k$-ideals of $R$ containing $P$. By maximality of $P$ either $P=(\bar{P}: A)_{l}$ or $\overline{(\bar{P}: A)_{l}} \cap M=(\bar{P}: A)_{l} \cap M \neq \emptyset$ as $(\bar{P}: A)_{l}$ is a $k$-ideal. Similarly $P=(\bar{P}: A)_{r}$ or $\overline{(\bar{P}: A)_{r}} \cap M=(\bar{P}: A)_{r} \bigcap M \neq \emptyset$ as $(\bar{P}: A)_{r}$ is a $k$-ideal. Suppose that $(\bar{P}: A)_{r} \cap M \neq \emptyset$. Let $x \in(\bar{P}: A)_{r} \cap M$. We consider two separate cases.

Case 1: $A \cap M \neq \emptyset$. Let $a \in A \cap M$. Since $x \in(\bar{P}: A)_{r}$ we have $\left\langle x>_{k} \subseteq(\bar{P}\right.$ : $A)_{r}$, since $(\bar{P}: A)_{r}$ is a $k$-ideal. Hence $A<x>_{k} \subseteq \bar{P}$. Since $a, x \in M$ and $M$ is an $m_{2}$-system of $R$ there exists $\left.a_{1} \in<a\right\rangle_{k}$ and there exists $\left.x_{1} \in<x\right\rangle_{k}$ such that $a_{1} x_{1} \in M$. Since $A<x>_{k} \subseteq \bar{P}$ implies $a_{1} x_{1} \in \bar{P}$. Therefore $a_{1} x_{1} \in \bar{P} \cap M$, which is impossible since $\bar{P} \cap M=\emptyset$. Thus $P=(\bar{P}: A)_{r}$ in this case. Similarly if $y \in(\bar{P}: A)_{l} \cap M$ then it follows that $P=(\bar{P}: A)_{l}$. Thus if $A \cap M \neq \emptyset$ then $(\bar{P}: A)_{r}=(\bar{P}: A)_{l}=P$.

Case 2: $A \cap M=\emptyset$. Again we have $A<x>_{k} \subseteq \bar{P}$. This implies that $A \subseteq\left(\bar{P}:<x>_{k}\right)_{l}=P$ by case 1 . This contradicts our assumption that $A \nsubseteq \subseteq$. Thus $(\bar{P}: A)_{l}=P$ in this case. Similarly $(\bar{P}: A)_{r}=P$. Finally we show that $P$ is 2-prime. Let $A$ and $B$ are $k$-ideals of $R$ such that $A B \subseteq P$ and $A \nsubseteq P$. Clearly $P \subseteq \bar{P}$. Hence $A B \subseteq \bar{P}$. It follows that $B \subseteq(\bar{P}: A)_{r}=P$. Therefore $B \subseteq P$.

Theorem 1.17. Let $Q$ be an ideal of $R$ and let $M$ be an $m_{0}$-system of $R$ such that $Q \cap M=\emptyset$. then there exists a 0-prime ideal $P$ of $R$ such that $Q \subseteq P$ with $P \cap M=\emptyset$. 
The proof is similar to Theorem 1.16.

Definition 1.18. If $R$ is a semiring and $Q$ is an ideal of $R$ then $Q$ is 0-(2-) semiprime ideal if $A$ is an ideal ( $k$-ideal) of $R$ such that $A^{2} \subseteq Q$ implies $A \subseteq Q$. If $R$ is semiring and $Q$ is an ideal of $R$ then $Q$ is 1-semirprime if $A$ is an ideal of $R$ such that $\bar{A} A \subseteq Q$ implies $A \subseteq Q$.

Definition 1.19. $N \subseteq R$ is called an $n_{0}$-system if for every $a \in N$ there exists $x \in R$ such that $a x a \in N . N \subseteq R$ is called an $n_{1}$-system if for every $a \in N$ there exists $a_{1} \in\langle a\rangle_{k}$ and there exists $a_{2} \in<a>$ such that $a_{1} a_{2} \in N . N \subseteq R$ is called an $n_{2}$-system if for every $a \in N$ there exists $a_{1}, a_{2} \in<a>_{k}$ such that $a_{1} a_{2} \in N$.

Lemma 1.20. If $S$ is a 0-semiprime ideal of $R$ then $S$ is a 2-semiprime ideal(1semiprime ideal) of $R$.

But the converse need not be true as the following an example shows.

Example 1.21. Consider the semiring in Example 1.10 Clearly $S=\{0,3\}$ is 2semiprime ideal but not 0 -semiprime ideal. Since if $A=\{0,2,3\}$, then $A$ is an ideal and $A^{2}=\{0,3\} \subseteq S$ but $A \nsubseteq S$.

Theorem 1.22. If $S$ is a k-ideal of $R$, then $S$ is 0-semiprime ideal iff $S$ is 2semirpime ideal.

The proof is similar to Theorem 1.11.

Lemma 1.23. Let $A$ be an $n_{1}$-system and $a \in A$. Then there is some $m_{1}$-system $M$ with $a \in M \subseteq A$.

Proof. Let $a \in A$. Hence there exists $\left.a_{1} \in<a\right\rangle_{k}$ and $\left.a_{2} \in<a\right\rangle$ such that $a_{1} a_{2} \in A$. Since $a_{1} a_{2} \in A$ there exists $a_{1}^{\prime} \in<a_{1} a_{2}>_{k}$ and $a_{2}^{\prime} \in<a_{1} a_{2}>$ such that $a_{1}^{\prime} a_{2}^{\prime} \in A$. Continuing this process, we get a sequence $\left\{a, a_{1} a_{2}, a_{1}^{\prime} a_{2}^{\prime}, a_{1}^{\prime \prime} a_{2}^{\prime \prime}, \ldots\right\}$ such that for every positive integer $k, a_{1}^{k} a_{2}^{k} \in A$ with $<a>_{k} \supseteq<a_{1} a_{2}>_{k} \supseteq<$ $a_{1}^{\prime} a_{2}^{\prime}>_{k} \supseteq<a_{1}^{\prime \prime} a_{2 \prime \prime}^{\prime \prime}>_{k} \supseteq \ldots$ and $<a>\supseteq<a_{1} a_{2}>\supseteq<a_{1}^{\prime} a_{2}^{\prime}>\supseteq \ldots$ Take $M=$ $\left\{a, a_{1} a_{2}, a_{1}^{\prime} a_{2}^{\prime}, a_{1}^{\prime \prime} a_{2}^{\prime \prime}, \ldots\right\}$. We show that $M$ is a desired $m_{1}$-system. If $a_{1}^{l} a_{2}^{l}, a_{1}^{k} a_{2}^{k} \in M$ (w.l.o.g.,let $k \leq l$ ) then $<a_{1}^{k} a_{2}^{k}>_{k} \supseteq<a_{1}^{l} a_{2}^{l}>_{k}$ and $<a_{1}^{k} a_{2}^{k}>\supseteq<a_{1}^{l} a_{2}^{l}>$. Now there exists $a_{1}^{l+1} \in<a_{1}^{l} a_{2}^{l}>_{k}$ and there exists $a_{2}^{l+1} \in<a_{1}^{l} a_{2}^{l}>\subseteq<a_{1}^{k} a_{2}^{k}>$ such that $a_{1}^{l+1} a_{2}^{l+1} \in M$. This implies that $M$ is a desired $m_{1}$-system.

Lemma 1.24. Let $A$ be an $n_{0}$-system and $a \in A$. Then there is some $m_{0}$-system $M$ with $a \in M \subseteq A$.

Lemma 1.25. Let $A$ be an $n_{2}$-system and $a \in A$. Then there is some $m_{2}$-system $M$ with $a \in M \subseteq A$.

Definition 1.26. If $A$ is an ideal of $R$, then we define $\mathcal{B}_{0}(A)=\bigcap\{P$ is a 0-prime ideal of $R$ and $A \subseteq P$ \}. Similarly we define $\mathcal{B}_{1}(A)$ and $\mathcal{B}_{2}(A)$.

Theorem 1.27. Let $Q$ be an ideal of $R$. (i) $\bar{Q}$ is a 2-semiprime ideal in $R$ iff $\mathcal{B}_{2}(\bar{Q})$ 
$=\bar{Q}$. (ii) $\bar{Q}$ is a 1-semiprime ideal in $R$ iff $\mathcal{B}_{1}(\bar{Q})=\bar{Q}$. (iii) $Q$ is a 0 -semiprime ideal in $R$ iff $\mathcal{B}_{0}(Q)=Q$.

Proof. Suppose $\mathcal{B}_{2}(\bar{Q})=\bar{Q}$. Then $\bar{Q}$ is the intersection of the 2-prime ideals of $R$ which contain $Q$ from which it follows easily that $\bar{Q}$ is 2 -semiprime. Conversely, let $\bar{Q}$ be 2-semiprime. Clearly $\bar{Q} \subseteq \mathcal{B}_{2}(\bar{Q})$. Let $a \in R \backslash \bar{Q}$. Since $\bar{Q}$ is 2-semiprime, we have $R \backslash \bar{Q}$ is an $n_{2}$-systme of $R$. By Lemma 1.25 there exists an $m_{2}$-system $M$ in $R$ such that $a \in M \subseteq R \backslash \bar{Q}$. By Theorem 1.16 there exists a 2-prime ideal $P$ of $R$ such that $Q \subseteq P$ and $P \bigcap M=\emptyset$. Then $a \notin P$ and so $a \notin \mathcal{B}_{2}(\bar{Q})$. Thus $\bar{Q}=\mathcal{B}_{2}(\bar{Q})$ and the proof is complete.

The next is a direct consequence of the above theorem.

Corollary 1.28([1], Theorem 1). Let $R$ be a semiring. Then the following assertions are equivalent: $1 . R$ is fully idempotent. 2. Each proper ideal of $R$ is the intersection of prime ideals which contain it.

\section{References}

[1] J. Ahsan, Fully idempotent semirings, Proc. japan Acad., 69, Ser.A(1993), 185-188.

[2] F.E.Alarcon and D. Polkowska, Fully Prime Semirings, Kyungpook Math. J., 40(2000), 239-245.

[3] P. Dheena and P. Nandakumar, On regular semirings, Indian Journal of Mathematics, 45(2)(2003), 135-140.

[4] N. H. McCoy, Theory of Rings, Macmillan, New York, 1964

[5] M.K.Sen and M.R.Adhikari, On maximal k-ideals of semirings, Proc.Amer. Math.Soc., 118(3), July 1993, 699-703.

[6] M.K.Sen and P.Mukhopadhyay, Von Neumannian Regularity in Semirings, Kyungpook Math.J., 35(1995), 249-258. 